\title{
STRUCTURE AND COMPOSITION OF TWO TRANSITIONAL CIRCUMSTELLAR DISKS IN CORONA AUSTRALIS
}

\author{
A. M. Hughes ${ }^{1}$, S. M. Andrews ${ }^{1}, 11$, D. J. Wilner ${ }^{1}$, M. R. Meyer ${ }^{2}$, J. M. Carpenter ${ }^{3}$, C. Qi ${ }^{1}$, A. S. Hales ${ }^{4}$, S. Casassus ${ }^{5}$,

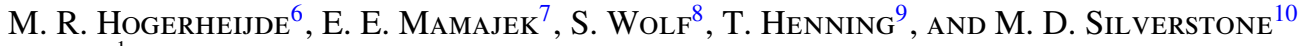 \\ ${ }^{1}$ Harvard-Smithsonian Center for Astrophysics, 60 Garden Street, Cambridge, MA 02138, USA; \\ mhughes@cfa.harvard.edu, sandrews@cfa.harvard.edu, dwilner@cfa.harvard.edu, cqi@cfa.harvard.edu \\ ${ }^{2}$ Institute for Astronomy ETH, Physics Department, HIT J 22.4, CH-8093 Zurich, Switzerland; mmeyer@phys.ethz.ch \\ ${ }^{3}$ California Institute of Technology, Department of Astronomy, MC 105-24, Pasadena, CA 91125, USA; jmc @ astro.caltech.edu \\ ${ }^{4}$ National Radio Astronomy Observatory, 520 Edgemont Road, Charlottesville, VA 22903-2475, USA; ahales@alma.cl \\ ${ }^{5}$ Departamento de Astronomía, Universidad de Chile, Casilla 36-D, Santiago, Chile; simon@ das.uchile.cl \\ ${ }^{6}$ Leiden Observatory, Leiden University, P.O. Box 9513, 2300 RA Leiden, The Netherlands; michiel@ strw.leidenuniv.nl \\ ${ }^{7}$ Department of Physics \& Astronomy, University of Rochester, Rochester, NY 14627-0171, USA; emamajek@ pas.rochester.edu \\ ${ }^{8}$ Institute of Theoretical Physics and Astrophysics, University of Kiel, Leibnizstrasse 15, 24098 Kiel, Germany; wolf@ astrophysik.uni-kiel.de \\ ${ }^{9}$ Max-Planck Institute for Astronomy, Koenigstuhl 17, D-69117 Heidelberg, Germany; henning@ @pia-hd.mpg.de \\ ${ }^{10}$ Eureka Scientific, Inc., 113 Castlefern Drive, Cary, NC 25713, USA; mdsilverstone@ att.net \\ Received 2010 February 27; accepted 2010 July 18; published 2010 August 13
}

\begin{abstract}
The late stages of evolution of the primordial circumstellar disks surrounding young stars are poorly understood, yet vital to constraining theories of planet formation. We consider basic structural models for the disks around two $\sim 10$ Myr old members of the nearby RCrA association: RX J1842.9-3532 and RX J1852.3-3700. We present new arcsecond-resolution maps of their $230 \mathrm{GHz}$ continuum emission from the Submillimeter Array and unresolved $\mathrm{CO}(3-2)$ spectra from the Atacama Submillimeter Telescope Experiment. By combining these data with broadband fluxes from the literature and infrared fluxes and spectra from the catalog of the Formation and Evolution of Planetary Systems Legacy program on the Spitzer Space Telescope, we assemble a multiwavelength data set probing the gas and dust disks. Using the Monte Carlo radiative transfer code RADMC to model simultaneously the spectral energy distribution and millimeter continuum visibilities, we derive basic dust disk properties and identify an inner cavity of radius $16 \mathrm{AU}$ in the disk around RX J1852.3-3700. We also identify an optically thin 5 AU cavity in the disk around RX J1842.9-3532, with a small amount of optically thick material close to the star. The molecular line observations suggest an intermediate disk inclination in RX J1842.9-3532, consistent with the continuum emission. In combination with the dust models, the molecular data allow us to derive a lower CO content than expected, suggesting that the process of gas clearing is likely underway in both systems, perhaps simultaneously with planet formation.
\end{abstract}

Key words: circumstellar matter - protoplanetary disks - stars: individual (RX J1842.9-3532, RX J1852.3-3700) - stars: pre-main sequence

Online-only material: color figures

\section{INTRODUCTION}

One of the key problems in planet formation is understanding how the reservoir of planet-forming material-the disk of gas and dust around a young star-evolves with time. Perhaps the most compelling stage in the evolution of protoplanetary disks is the "transitional" stage during which gas and dust are cleared from the system (e.g., Strom et al. 1989; Skrutskie et al. 1990). This stage appears to be either rapid or rare, since fewer than $10 \%$ of low- to intermediate-mass stars in young star-forming regions are typically observed to be transitional systems (e.g., Silverstone et al. 2006; Cieza et al. 2007; Uzpen et al. 2008), perhaps with some dependence on stellar mass (e.g., Currie \& Sicilia-Aguilar 2010). Transition disks are traditionally identified empirically by a deficit of mid-infrared (mid-IR) dust excess over the stellar photosphere relative to comparable young systems. This deficit is associated with a lack of hot dust close to the star. The advent of the Spitzer Space Telescope has revolutionized the quality and quantity of available data on the dust content of young stellar systems, particularly transitional objects (see, e.g., Najita et al. 2007; Currie et al.

\footnotetext{
${ }^{11}$ Hubble Fellow.
}

2009; Muzerolle et al. 2010). One of its many contributions has been to reveal a new class of gapped or "pre-transitional" disks, in which an anomalously faint mid-IR spectrum is bracketed by substantial near- and far-IR excesses (Espaillat et al. 2007, 2008, 2010). Follow-up of Spitzer-identified transitional systems with high spatial resolution observations of continuum emission at millimeter wavelengths has led to the confirmation that mid-IR spectral deficits are associated with a lack of long-wavelength emission from the disk center (Calvet et al. 2002, 2005; Hughes et al. 2007, 2009; Brown et al. 2007, 2008, 2009; Piétu et al. 2007; Espaillat et al. 2008). The properties of systems with gaps and holes are beginning to provide valuable insight into the physical mechanism(s) responsible for the dispersal of the circumstellar disk. Photophoresis, the presence of unknown binary companions, grain growth, dynamical interactions with giant planets in formation, and photoevaporation have all been suggested as clearing mechanisms; there is some indication that different processes may dominate at different ages (see, e.g., Sicilia-Aguilar et al. 2010).

The Formation and Evolution of Planetary Systems (FEPS) Legacy program on the Spitzer Space Telescope (Meyer et al. 2006; Carpenter et al. 2008) is a spectrophotometric survey 
of nearby Sun-like stars, with masses from 0.7 to $1.3 M_{\odot}$ and ages between $3 \mathrm{Myr}$ and $3 \mathrm{Gyr}$. These ages bracket the period of time when gas and dust were cleared from the primordial solar nebula, and the epoch when the solar system achieved its present configuration. For 309 objects in the FEPS sample, the survey includes IRAC 3.6-8.0 $\mu \mathrm{m}$ photometry to probe for hot, dusty analogs to the asteroid belt in the solar system, MIPS 24 and $70 \mu \mathrm{m}$ photometry to probe dust in the Kuiper Belt regions, and InfraRed Spectrograph (IRS) 5-40 $\mu \mathrm{m}$ spectra to search for mineralogical features. The excellent coverage of the IR spectral energy distribution (SED) permits modeling of the temperature, size, composition, and an initial estimate of the spatial distribution of dust grains (see, e.g., Kim et al. 2005; Bouwman et al. 2008; Cortes et al. 2009). The IRS spectra are particularly useful for identifying systems with inner cavities or gaps in their dust distribution. However, there are many degeneracies inherent in the derivation of spatial information from unresolved spectra, and the SED provides little information about the large grains that comprise most of the dust mass in the system. It is therefore desirable to combine the information from the SED with spatially resolved observations at millimeter wavelengths. Spectral line observations of low-level rotational emission from the $\mathrm{CO}$ molecule can provide a complementary probe of the molecular gas content, which is the dominant mass constituent, and can yield important clues to the evolution of transitional objects.

In this paper, we analyze the SEDs and resolved millimeter continuum emission of two sources from the FEPS sample: RX J1842.9-3532 and RX J1852.3-3700. These sources were detected in the ROSAT All-Sky Survey and identified as young stars by Neuhäuser et al. (2000). They have spectral type K2 and K3, respectively (Carpenter et al. 2008), and have been classified as classical T Tauri stars (cTTSs) based on the presence of strong $\mathrm{H} \alpha$ emission lines. Neither was identified as a multiple-star system in the speckle-interferometric observations of Köhler et al. (2008). They are located within a few degrees of the CrA molecular cloud (distance 130 pc; Neuhäuser et al. 2000), and have kinematics and secular parallaxes consistent with the RCrA association (E. Mamajek 2010, private communication). The dust grain composition of the inner disks of both systems was modeled in detail by Bouwman et al. (2008), and Pascucci et al. (2007) report the detection of [Ne II] emission likely arising from a hot disk atmosphere and calculate accretion rates for both systems of order $10^{-9} M_{\odot} \mathrm{yr}^{-1}$. The estimated stellar ages are $\sim 10 \mathrm{Myr}$, among the oldest in the $1-10 \mathrm{Myr}$ range for T Tauri stars in CrA measured by Neuhäuser et al. (2000). These sources were selected for study on the basis of their age, their brightness in the $1.2 \mathrm{~mm}$ continuum (Carpenter et al. 2005), and their accessibility to the Submillimeter Array (SMA), ${ }^{12}$ so that the spectral information from the FEPS survey could be combined with resolved submillimeter observations. They were also chosen for their proximity on the sky, which increases the efficiency of submillimeter observations by allowing them to share calibrators. The high-quality Spitzer IRS spectra provide constraints on the dust composition and temperature structure on the two systems, and both exhibit a flux deficit in the mid-IR photospheric excess that points to the presence of an inner hole or gap.

Sections 2 and 3 describe the collection of new data from the SMA and the Atacama Submillimeter Telescope Experiment

\footnotetext{
12 The Submillimeter Array is a joint project between the Smithsonian Astrophysical Observatory and the Academia Sinica Institute of Astronomy and Astrophysics and is funded by the Smithsonian Institution and the Academia Sinica.
}

(ASTE) that complement the spectra and broadband fluxes from the FEPS survey and the literature. In Section 4.1 we present the tools and techniques that we use to model the SED and resolved millimeter-wavelength data, and we present the models of the dust disk structures in Section 4.2. In Section 4.3, we explore the dust disk model in the context of the constraints on the gas content of the two systems. We summarize our results and discuss their implications in Section 5.

\section{OBSERVATIONS AND DATA REDUCTION}

\subsection{SMA Observations}

The SMA observations of the two sources took place on 2005 May 14 during a full six-hour track with six of the $6 \mathrm{~m}$ diameter antennas operating in the compact-north configuration, yielding baseline lengths between 10 and $180 \mathrm{~m}(8$ and $140 \mathrm{k} \lambda)$. The phase stability was adequate for most of the track, with phase differences of $20^{\circ}-30^{\circ}$ between calibrator scans, but the phases lost coherence during the last hour of the night. The weather was fair, with the $225 \mathrm{GHz}$ atmospheric opacity increasing from 0.10 to 0.14 throughout the night. Observations of the two FEPS sources were alternated with observations of the quasar J1924-292 at 15 minute intervals to calibrate the atmospheric and instrumental variations of phase and amplitude gain. Callisto was used as the flux calibrator, with a calculated brightness of $6.59 \mathrm{Jy}$; the derived flux of J1924-292 was 5.4 Jy, with an estimated uncertainty of $\lesssim 20 \%$. The correlator was configured to provide a spectral resolution of 512 channels over the $104 \mathrm{MHz}$ bandwidth in the chunk containing the $\mathrm{CO}(2-1)$ line, corresponding to a velocity resolution of $0.26 \mathrm{~km} \mathrm{~s}^{-1}$. Two other $104 \mathrm{MHz}$ chunks were observed at high resolution at frequencies corresponding to the ${ }^{13} \mathrm{CO}(2-1)$ and $\mathrm{C}^{18} \mathrm{O}(2-1)$ lines. The remainder of bandwidth in each $2 \mathrm{GHz}$ wide sideband was devoted to measuring the $230 \mathrm{GHz}$ continuum, observed at a spectral resolution of $4.2 \mathrm{~km} \mathrm{~s}^{-1}$. The data were calibrated using the MIR software package, ${ }^{13}$ and imaging was carried out with the MIRIAD software package.

\subsection{ASTE Observations}

Observations of both FEPS sources took place on 2008 June 28 and 29 using the CATS345 receiver on the $10.4 \mathrm{~m} \mathrm{ASTE}$ dish. RX J1842.9-3532 was observed on both nights, while RX J1852.3-3700 was observed only on the second night. The receiver was tuned to place the $\mathrm{CO}(3-2)$ rotational transition in the lower sideband with the $\mathrm{HCO}^{+}(4-3)$ transition in the upper sideband. The high-resolution spectrometer mode was used to partition the $128 \mathrm{MHz}$ bandwidth into 1024 channels, yielding a spectral resolution of $0.11 \mathrm{~km} \mathrm{~s}^{-1}$. Position switching was used to subtract the instrumental and sky background. In order to ensure that the detected $\mathrm{CO}$ emission originated at the position of the star, we used an offset position 1.5 to the east on the night of June 28 and 1.5 to the west on June 29 and averaged the baseline-subtracted spectra to create the final spectrum. The double-peaked CO(3-2) line from RX J1842.9-3532 is detected independently on both nights using the different offset positions, which allows us to localize the emission to within 1.5 of the star ( $\sim 4$ beam widths).

The $\mathrm{CO}(3-2)$ and $\mathrm{HCO}^{+}(4-3)$ tuning of the receiver resulted in a spurious $1.11 \mathrm{MHz}$ sinusoidal ripple of variable amplitude and phase across the bandpass, which was subtracted individually from each $10 \mathrm{~s}$ integration in the following manner. The

\footnotetext{
13 http://cfa-www.harvard.edu/ cqi/mircook.html
} 

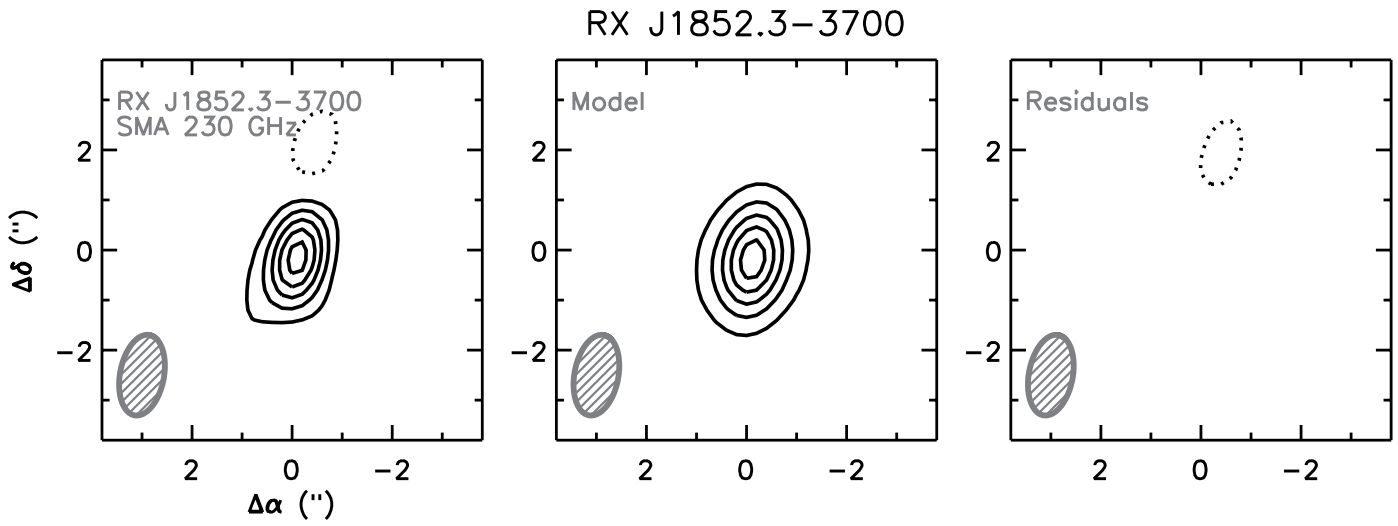

RX J1842.9-3532
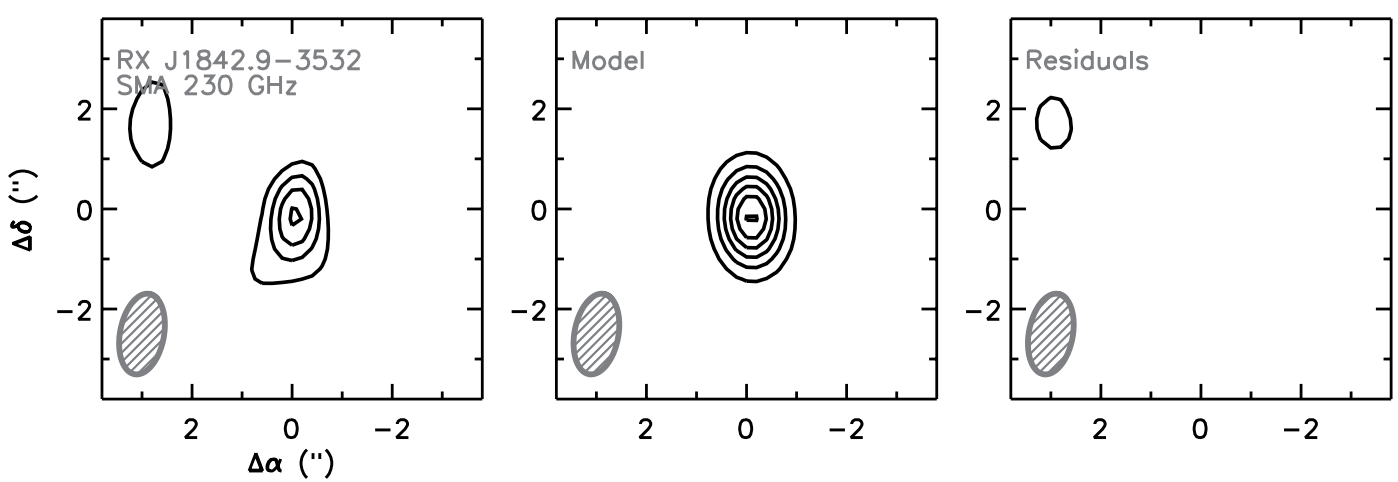

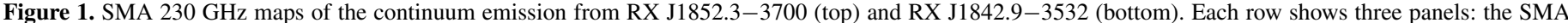

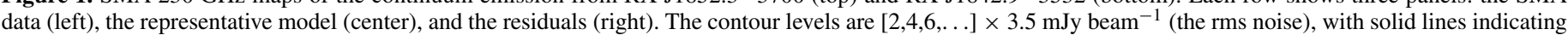
positive contours and dotted line indicating negative contours. The $1^{\prime \prime} .0 \times 11^{\prime \prime} .7$ synthesized beam is indicated by the ellipse in the lower left corner.

amplitude and frequency of the ripple were estimated by finding the peak in a fast Fourier transform of the spectrum, and then a least-squares fit was performed to determine the precise amplitude, phase, and frequency of the sinusoidal ripple, plus the slope and intercept of a linear component to remove the worst of the baseline features. During this process, the region of the spectrum containing the line was not included in the fit so as to avoid inadvertently subtracting it. Integrations with an abnormally large ripple amplitude or highly irregular baseline shape across the bandpass were discarded (roughly $10 \%$ of each data set). After subtracting the sinusoid and linear fit, a third-order polynomial was subtracted from each integration using the CLASS software package, ${ }^{14}$ fitting the $20 \mathrm{~km} \mathrm{~s}^{-1}$ to each side of the region that appeared to contain the line. The $10 \mathrm{~s}$ integrations were then averaged together to produce a spectrum for each night; the spectra for each night were averaged and weighted according to their total integration time $\left(\sqrt{1 / t_{\text {int }}}\right)$ to produce the final spectrum. Due to differing exposure times and poorer weather on the night of June 29, the rms noise in the spectrum of RX J1842.9-3532 is $24 \mathrm{mK}$, while the rms noise in the spectrum of RX J1852.3-3700 is $39 \mathrm{mK}$. To derive the absolute flux scale, we calculated the main beam efficiency using observations of the calibrator M17SW taken throughout the night. Assuming a peak main beam temperature in the $\mathrm{CO}(3-2)$ line of $85.3 \mathrm{~K}$, derived on the CSO $10.4 \mathrm{~m}$ telescope by Wang et al. (1994), we derive main beam efficiencies that varied between 0.55 and 0.63 over the course of the two nights.

\footnotetext{
${ }^{14} \mathrm{http}: / /$ www.iram.fr/IRAMFR/GILDAS
}

\section{RESULTS}

\subsection{Millimeter Continuum}

With the SMA observations, we detect $230 \mathrm{GHz}$ continuum emission from the outer disks around both target stars. The contour maps in the left panels of Figure 1 show the strong detection of emission centered on the star position at the middle of the field. The spatial resolution of the data is $1^{\prime \prime} .0 \times 1^{\prime \prime} .7$, or $\sim 150 \mathrm{AU}$ at a distance of $130 \mathrm{pc}$, well matched to the typical size of a circumstellar disk but insufficient to provide evidence for the presence or absence of an inner cavity. In order to estimate the integrated flux and approximate outer disk geometry, a Gaussian fit to the visibilities was performed using the MIRIAD task uvfit. For RX J1842.9-3532, the fit yields an integrated flux of $49 \pm 8 \mathrm{mJy}$ and indicates that the disk is only marginally resolved: the major and minor axes lengths of $0.74 \pm 0.32$ and $0 . .44 \pm 0$ '.32 suggest that the disk is probably not viewed face-on, but do not place strong constraints on the inclination. We estimate an inclination angle of $54^{\circ}$ based on these measurements, which is consistent with the morphology of the ASTE $\mathrm{CO}(3-2)$ spectrum described in Section 3.2. The fit to the RX J1852.3-3700 visibilities yields a flux of $60 \pm 8 \mathrm{mJy}$ and the major and minor axes of length $0.76 \pm 0.21$ and $0.73 \pm 0.21$, consistent with a nearly face-on geometry; we use these measurements to estimate an inclination of $16^{\circ}$. The inclination estimates are highly uncertain, but the intermediate geometry of RX J1842.9-3532 is supported by the line profile in Section 3.2 and the more face-on geometry of RX J1852.3-3700 is supported by the $\mathrm{H} \alpha$ line profile modeling of Pascucci et al. (2007). While 

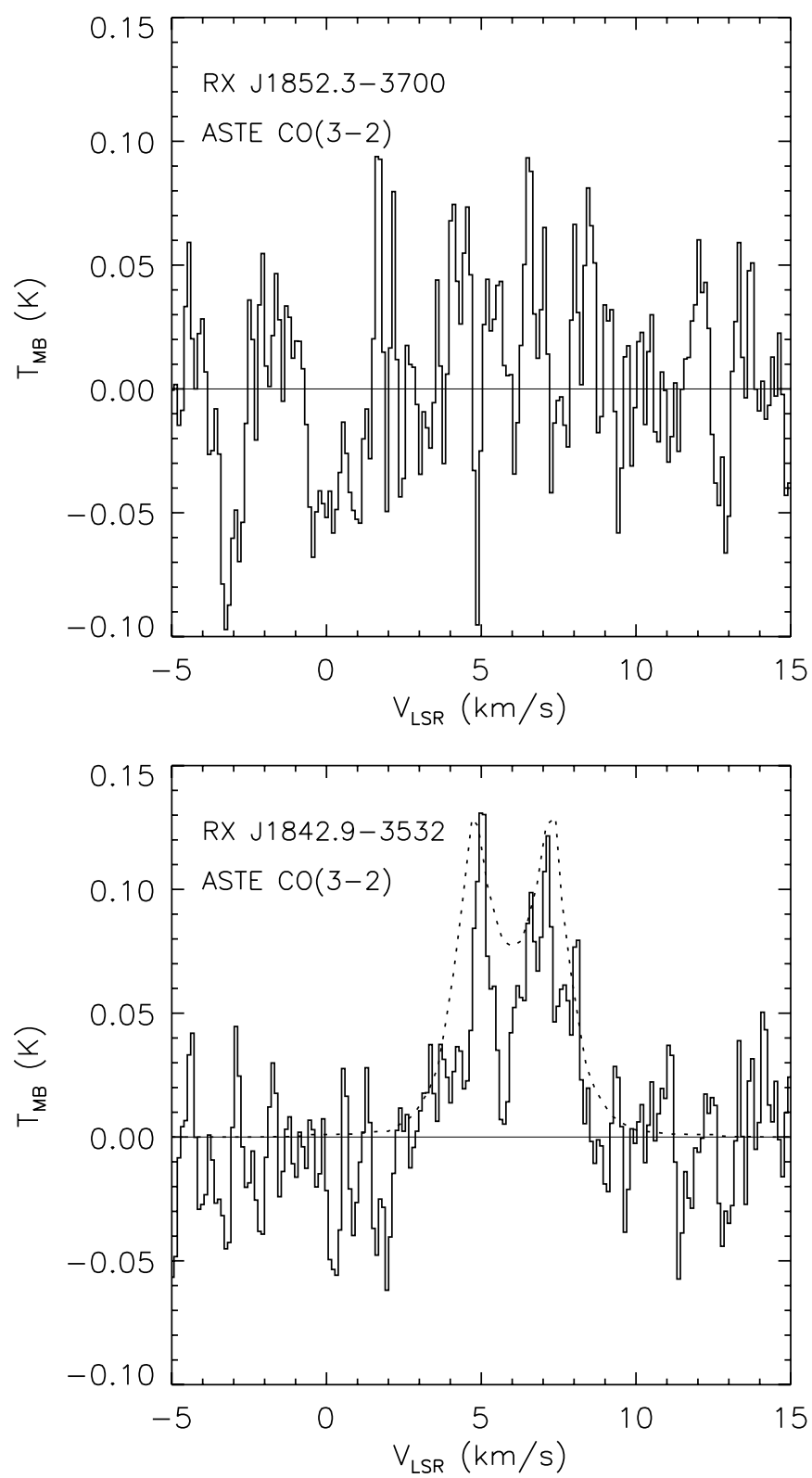

Figure 2. ASTE CO(3-2) spectra of the disks around RX J1852.3-3700 (upper) and RX J1842.9-3532 (lower). No emission is detected from the RX J1852.3-3700 system. The RX J1842.9-3532 emission (solid line) displays the characteristic double-peaked profile characteristic of an inclined structure in Keplerian rotation about the central star. The line profile predicted by the SEDand visibility-based model of the dust disk structure (dotted line) compares favorably with the observations.

observations at higher resolution would be advantageous for constraining the detailed mass distribution, even the rudimentary estimates of disk geometry provided by these observations are useful for constraining the disk properties when combined with constraints from the broadband SED. Simultaneous modeling of the SED and millimeter-wavelength visibilities is described in Section 4.1.

\section{2. $\mathrm{CO}(2-1)$ and $\mathrm{CO}(3-2)$ Line Observations}

We do not detect molecular gas emission from either system in the interferometric SMA observations of the $\mathrm{CO}(2-1)$, ${ }^{13} \mathrm{CO}(2-1)$, or $\mathrm{C}^{18} \mathrm{O}(2-1)$ lines. The data provide a $3 \sigma$ upper limit of $0.4 \mathrm{Jy} \mathrm{beam}^{-1}$ in each $0.26 \mathrm{~km} \mathrm{~s}^{-1}$ channel, with a synthesized beam size of $1^{\prime \prime} .5 \times 00^{\prime} .8$. Although the disks are only marginally resolved in the continuum emission, there is reason to expect that the extent of $\mathrm{CO}(2-1)$ emission may be several times larger than that of the continuum (see, e.g., Hughes et al. 2008b). As a result, spatial filtering may be a factor in the non-detection (see further discussion in Section 4.3).

We do not detect any $\mathrm{CO}(3-2)$ emission in the ASTE observations of the disk around RX J1852.3-3700, with an rms of $39 \mathrm{mK}$ in each $0.1 \mathrm{~km} \mathrm{~s}^{-1}$ channel. Observations of the disk around RX J1842.9-3532 reveal a double-peaked line profile, shown as a solid black line in Figure 2. The integrated strength of the $\mathrm{CO}(3-2)$ line is $0.24 \mathrm{~K} \mathrm{~km} \mathrm{~s}^{-1}$ with a peak main-beam brightness temperature of $130 \mathrm{mK}$ and FWHM of $2.6 \mathrm{~km} \mathrm{~s}^{-1}$. The double-peaked profile is consistent with material in Keplerian rotation about the star, viewed at an intermediate inclination angle of $\sim 54^{\circ}$. We detect no emission from the CrA molecular cloud near the line in velocity space, although it is possible that absorption from the cloud in the vicinity of the disk might influence the line shape despite the sources' large distance from CrA cloud center. In Section 4.3, we investigate the relationship of the $\mathrm{CO}(3-2)$ emission to the dust properties, including implications for the disk geometry and gas-to-dust mass ratio.

\section{ANALYSIS}

In order to characterize the basic properties of the disks, we seek a model that can reproduce the observational features of each system. We assemble a data set that combines the millimeter-wavelength properties of the gas and dust described above with constraints from the broadband SED and IRS spectrum. We use the IRS spectrum and SED from the FEPS database (described in Carpenter et al. 2008), including optical, near-IR, and millimeter fluxes collected from the literature (Neuhäuser et al. 2000; Skrutskie et al. 2006; Carpenter et al. 2005). Figures 3 and 4 show the SED (black points) and the IRS spectrum (red line) in the left panel for each disk, alongside the SMA $230 \mathrm{GHz}$ visibilities (black points) in the right panel. In order to improve the signal-to-noise ratio of the plotted data, the visibilities have been deprojected (see, e.g., Lay et al. 1997) according to the disk geometry inferred in Section 3.1 and averaged in bins of $15 \mathrm{k} \lambda$. For a mathematical description of the abscissas of the visibility plots, refer to Hughes et al. (2008b).

\subsection{Modeling the SED and Millimeter Visibilities}

In an effort to reproduce these observations, we generated synthetic broadband SEDs, Spitzer IRS spectra, and millimeter continuum visibilities using the radiative transfer method and disk structure models described by Andrews et al. (2009). In these flared, axisymmetric disk structure models, the radial surface density profile is characterized by a similarity solution for viscous accretion disks, $\Sigma \propto\left(R_{c} / R\right)^{\gamma} \exp \left[-\left(R / R_{c}\right)^{2-\gamma}\right]$, where $R_{c}$ is a characteristic radius and the normalization is proportional to the disk mass (for simplicity, the radial index has been fixed to $\gamma=1$; Lynden-Bell \& Pringle 1974; Hartmann et al. 1998). Vertically, the densities are distributed as a Gaussian with a scale height that varies as a power law with radius, $H \propto R^{1+\psi}$, with scale height $H_{100}$ at a radius of $100 \mathrm{AU}$. This parametric definition of the vertical dust distribution is maintained to mimic the sedimentation of dust grains below the disk atmosphere (e.g., Dullemond \& Dominik 2004b); no attempt is made to iterate on the density structure to force the dust into vertical hydrostatic equilibrium. To model the cleared inner disks for these transitional sources, we scale down the 

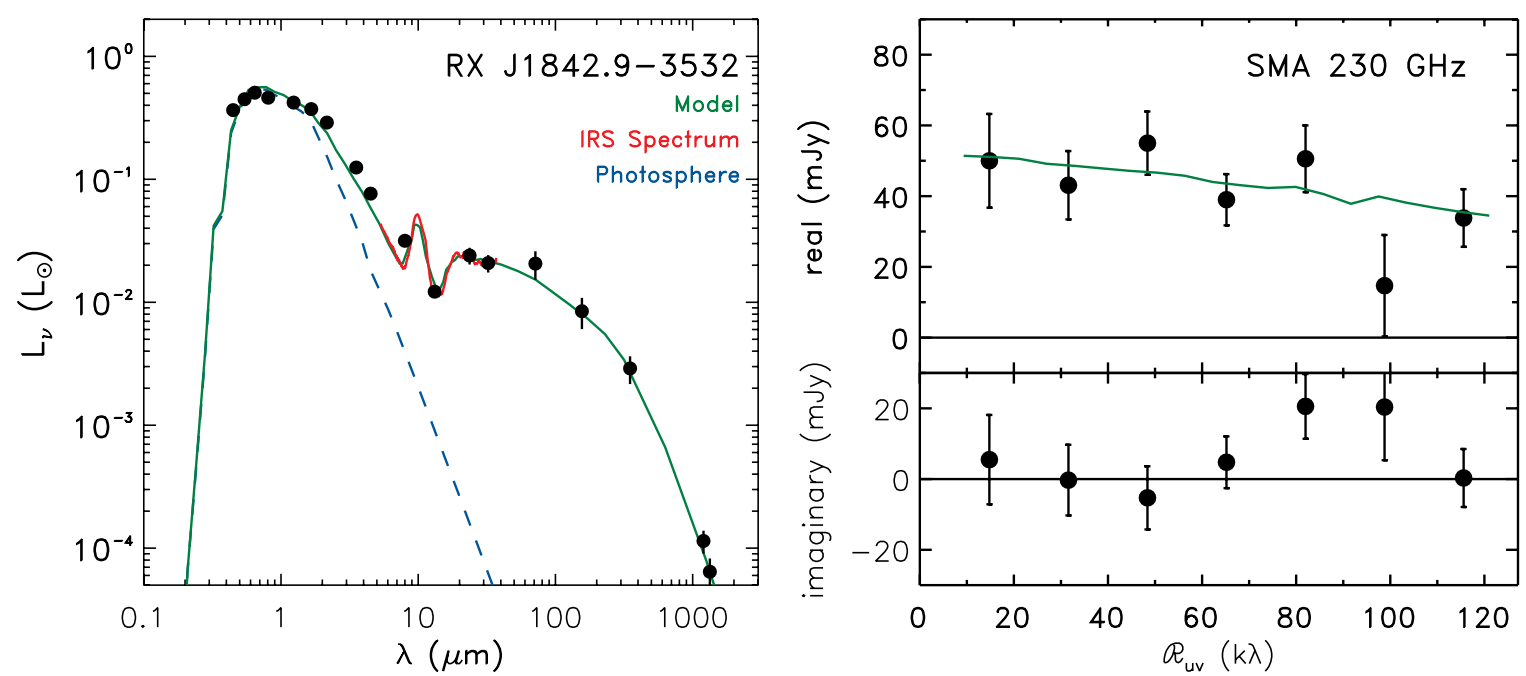

Figure 3. SED (left) and the real and imaginary components of the deprojected SMA $230 \mathrm{GHz}$ visibilities (right) for RX J1842.9-3532. The broadband SED (black points) and IRS spectrum (red line) are well reproduced by the best-fit RADMC disk structure model (green line). The model stellar photosphere (dashed blue line) is plotted for comparison. The units of the ordinate are defined so that $L_{v}=4 \pi d^{2} v F_{v}$ in units of $L_{\odot}$. For a mathematical definition of the abscissa, refer to Hughes et al. (2008b); the deprojection is carried out as in Lay et al. (1997).

(A color version of this figure is available in the online journal.)
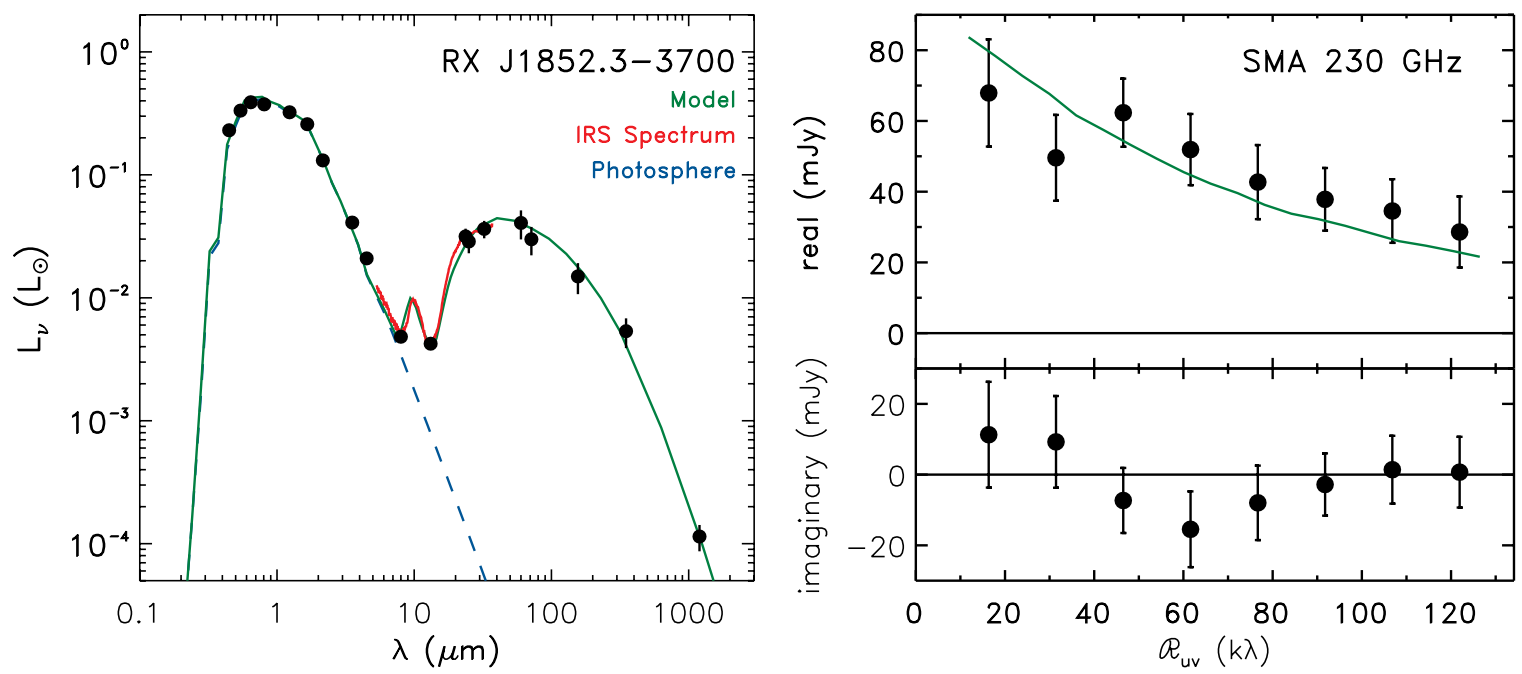

Figure 4. Same as Figure 3, but for RX J1852.3-3700.

(A color version of this figure is available in the online journal.)

surface densities by a factor $\delta_{\Sigma}$ inside a radius $R_{\text {cav }}\left(\Sigma_{\text {cav }}=\delta_{\Sigma} \Sigma\right.$; Andrews et al. 2009). Moreover, in an effort to better reproduce the detailed shape and solid state features in the IRS spectra, we permit a small (multiplicative) increase in the scale height at the cavity edge $\left(\delta_{H}\right)$ and adjust the dust grain properties in the inner disk (for details, see Andrews et al. 2010). Inner disk silicate opacities are taken from the Jena database (see, e.g., Ossenkopf \& Henning 1994).

For a given parametric disk structure, fixed input stellar information (Carpenter et al. 2008), and opacities (see Andrews et al. 2009), we use the two-dimensional Monte Carlo radiative transfer code RADMC (Dullemond \& Dominik 2004a) to calculate an internally consistent temperature structure and generate synthetic data products that can be compared to the observations. However, the parameter degeneracies introduced by the additional inner disk parameters and the high quality of the IRS spectra (requiring attention to mineralogy) make the minimization method described by Andrews et al. (2009) prohibitive. Instead, we aimed to find a representative model that can re- produce the basic features of the data by focusing on varying parameters such as the cavity size $\left(R_{\text {cav }}\right)$ and surface density reduction $\left(\delta_{\Sigma}\right)$. These models serve as initial estimates of the disk structures that can be refined when future observations are available (e.g., high angular resolution millimeter data).

\subsection{Representative Models}

Table 1 presents the parameters of representative disk structure models capable of reproducing the observational data for both systems, and indicates those parameters that were fixed by particular observational constraints. The $130 \mathrm{pc}$ distance to the RCrA association is from Neuhäuser et al. (2000) and the visual extinction and stellar properties are drawn from the FEPS database (Carpenter et al. 2008), while the inclination and position angle are estimated from the data as described in Sections 3.1 and 3.2. The other parameters are defined in Section 4.1; Andrews et al. $(2009,2010)$ include extensive discussion of the degeneracies between parameters and the ways in 

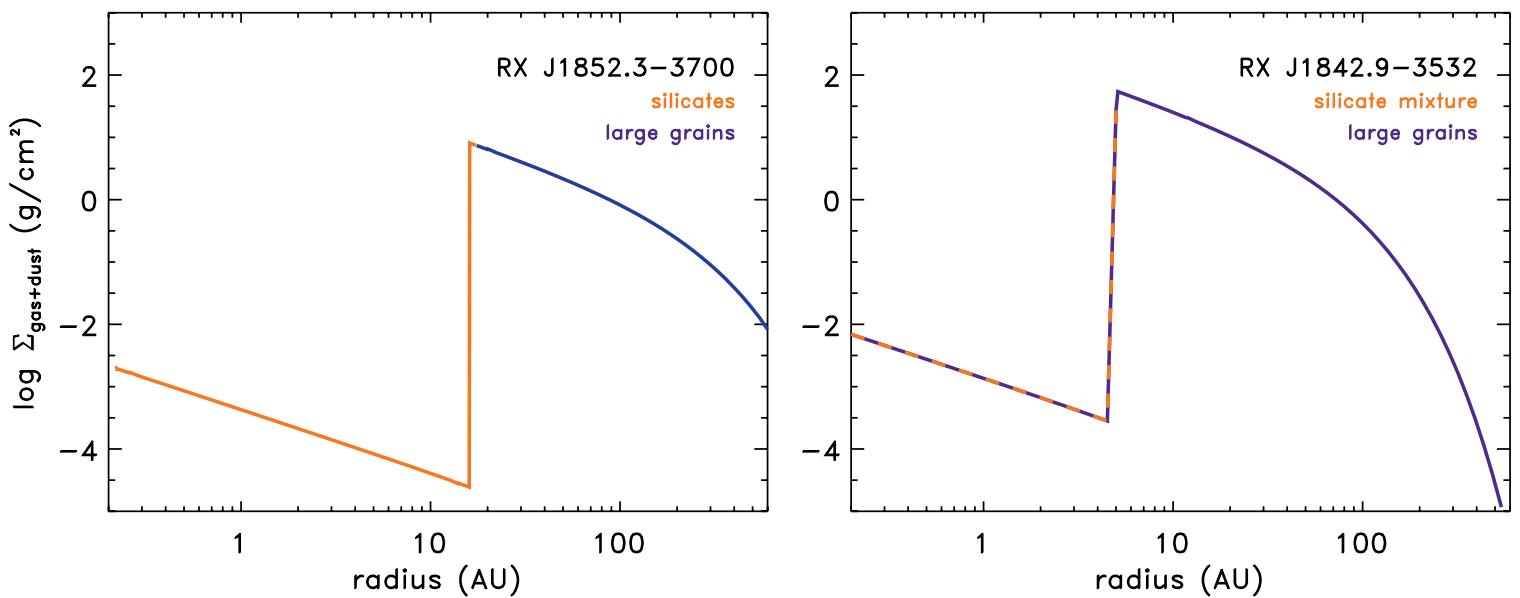

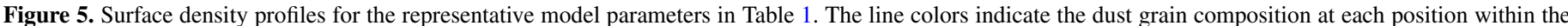

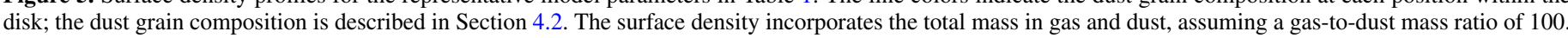
(A color version of this figure is available in the online journal.)

Table 1

Star and Estimated Disk Parameters

\begin{tabular}{lcc}
\hline \multicolumn{1}{c}{ Parameter } & RX J1842.9-3532 & RX J1852.3-3700 \\
\hline Distance $(\mathrm{pc})$ & 130 & 130 \\
$A_{\mathrm{V}}(\mathrm{mag})$ & 1.06 & 0.97 \\
Spectral type & $\mathrm{K} 2$ & $\mathrm{~K} 3$ \\
$\log g\left(\log \mathrm{cm} \mathrm{s}^{-2}\right)$ & 4.27 & 4.31 \\
$T_{\text {eff }}(\mathrm{K})$ & 4645 & 4854 \\
$i\left(^{\circ}\right)$ & 54 & 16 \\
P.A. $\left({ }^{\circ}\right)$ & 32 & -56 \\
$\gamma$ & 1.0 & 1.0 \\
\hline & Varied & 180 \\
\hline$R_{\mathrm{C}}(\mathrm{AU})$ & 50 & 0.016 \\
$M_{\mathrm{D}}\left(M_{\odot}\right)^{\mathrm{a}}$ & 0.010 & 0.2 \\
$\psi$ & 0.2 & 6.3 \\
$H_{100}(\mathrm{AU})$ & 4.8 & 16 \\
$R_{\text {cav }}(\mathrm{AU})$ & 5 & $3 \times 10^{-6}$ \\
$\delta_{\text {cav }}$ & $910^{-6}$ & 1.4 \\
$\delta H$ & 1 & \\
\hline
\end{tabular}

Note. ${ }^{\text {a }}$ Total mass in gas and dust, assuming a gas-to-dust mass ratio of 100 .

which the observational features are linked to the components of the disk structure model. Here we include comments on several parameters that are particularly relevant for reproducing the data described in this paper.

Inner disk structure $\left(R_{\mathrm{cav}}\right.$ and $\left.\delta_{\mathrm{cav}}\right)$. These parameters are tied primarily to the wavelength and magnitude of the rise in the IR flux longward of the $10 \mu \mathrm{m}$ silicate feature. It should be noted that while the density reduction $\delta_{\text {cav }}$ is greater for RX J1842.9-3532 than for RX J1852.3-3700, the initial difference in surface density must be taken into account: because RX J1842.9-3532 is almost a factor of 4 more compact than RX J1852.3-3700, the surface density throughout most of the disk, including within the cavity, is larger. As a result, the inner disk of RX J1852.3-3700 is entirely optically thin, while that of RX J1842.9-3532 includes both optically thick and optically thin regimes. The surface density profile of the two models is plotted in Figure 5. As indicated in Section 5, the details of the inner disk structure are not well constrained by these models, although the presence of an inner cavity of greatly reduced surface density is firmly indicated.

Puffing at the inner edge of the outer disk $(\delta H)$. The parameter $\delta H$, introduced in Andrews et al. (2010), describes the extent to which the scale height at the edge of the cavity is puffed up, which occurs because of the frontal illumination of the inner edge of the outer disk and is primarily manifested observationally in the shape of the far-IR SED. While a small $\delta H$ can help to account for the very steep mid- to far-IR jump in flux observed in the RX J1852.3-3700 IRS spectrum, no shadowing is required to reproduce the spectrum of RX J1842.9-3532.

Inner disk dust properties. We have very little information about the composition of dust grains in the outer disk, other than that millimeter-size grains must be present to account for the emission at long wavelengths. However, reproducing the features in the IRS spectrum requires us to vary the composition of the hot grains in the inner disk and wall that give rise to these features, as in Andrews et al. (2010). The $10 \mu \mathrm{m}$ silicate feature and steep rise in flux near $20 \mu \mathrm{m}$ from the disk around RX J1852.3-3700 are reproduced well by an inner disk and cavity edge populated entirely by small $(\sim 0.1 \mu \mathrm{m})$ amorphous silicate grains. For the disk around RX J1842.9-3532, the strength and position in wavelength of the silicate feature are well reproduced by a mixture of small $\left(a_{\max }=1 \mu \mathrm{m}\right)$ and large $\left(a_{\max }=1 \mathrm{~mm}\right)$ interstellar medium (ISM) composition grains $(\sim 80 \%)$ and crystalline and amorphous silicates $(\sim 20 \%)$ in the inner disk. This combination of grain compositions is by no means a unique solution to the problem of fitting the mid-IR spectrum, but merely demonstrates that a mixture of different grain properties is helpful in accounting for the observed spectral features. A more detailed mineralogical analysis of these systems can be found in Bouwman et al. (2008). The estimated mass of dust in the inner disk is $10^{-10} M_{\odot}$ for RX J1842.9-3532 and $10^{-6} M_{\odot}$ for RX J1852.3-3700.

The model SED and millimeter visibilities for the structural parameters in Table 1 are shown by the green lines in Figures 3 and 4 . They reproduce the basic features of all of the available dust disk diagnostics, including the broadband SED, the IRS spectrum, and the millimeter-wavelength visibilities. In the discussion below, we focus on the most robustly constrained model parameters, including the extent and surface density reduction of the inner cavity and the size and dust mass of the outer disk. 


\subsection{Constraints on Molecular Gas Content}

Here we compare the predictions of the dust disk model with the constraints on the CO emission described in Section 3.2. For simplicity, we assume that gas and dust are well mixed, with identical spatial and temperature distributions. We use the standard assumptions of a gas-to-dust mass ratio of 100:1 and a $\mathrm{CO}$ abundance of $10^{-4}$ relative to $\mathrm{H}_{2}$. To account for the depletion of gas-phase $\mathrm{CO}$ onto dust grains at low temperatures, we decrease the $\mathrm{CO}$ abundance by an additional factor of $10^{-4}$ in regions with temperatures $\leqslant 20 \mathrm{~K}$. As in Andrews et al. (2009), we then use the Monte Carlo molecular line radiative transfer code RATRAN (Hogerheijde \& van der Tak 2000) to calculate the level populations and predict the sky-projected intensity of $\mathrm{CO}$ arising from each system, given the underlying structure of the representative models derived in Section 4.2. We use the MIRIAD task convol to convolve the resulting intensity distributions with the $21^{\prime \prime} .1$ beam of the $10.4 \mathrm{~m}$ ASTE telescope, since the ASTE spectra provide the most stringent limits on the $\mathrm{CO}$ emission from the systems.

With the standard assumptions of gas-to-dust and $\mathrm{CO}$ to- $\mathrm{H}_{2}$ ratios, the model projection of $\mathrm{CO}(3-2)$ line flux strongly overpredicts both the upper limit for the disk around RX J1852.3-3700 and the detection of $\mathrm{CO}(3-2)$ emission from the disk around RX J1842.9-3532 by a factor of 4.8. In order for the model to successfully reproduce the weak emission from RX J1842.9-3532, the volume density of CO must be decreased to $8 \% \pm 3 \%$ of its initial value. A model spectrum for this case is given by the dotted line in Figure 2, and compares well with the strength and width of the observed $\mathrm{CO}$ spectrum. While the line peaks appear narrower than the model, the noise in the line is too large to merit modeling the profile in detail; it is also possible that contamination from remnant molecular cloud material could contribute to the narrowing of the peaks.

It is worthwhile to consider whether the apparently faint $\mathrm{CO}$ emission from these disks could be produced by overestimated model gas temperatures, rather than low $\mathrm{CO}$ abundances. In our models, we have assumed that the gas and dust are well mixed. However, the IR emission from these sources is too faint to accommodate a dust distribution in vertical hydrostatic equilibrium: the dust has apparently settled toward the disk midplane. The gas, on the other hand, should still feel a vertical pressure gradient, and therefore have a larger scale height than the dust (e.g., Dullemond \& Dominik 2004b; D'Alessio et al. 2006). As a result the gas in our model, which is assumed to follow the spatial distribution of the dust, is at somewhat lower temperatures than it would be in a thicker disk. This difference in temperature due to the physical location of the gas and dust is apparently exacerbated by exposure to high-energy (FUV, X-ray) radiation from the central star (Qi et al. 2006). Simulations show that these heating processes typically raise the gas temperature over that of the dust in the upper disk layers from which the CO emission arises (e.g., Kamp \& Dullemond 2004; Woitke et al. 2009). If anything, our model assumptions should then bias the results toward underestimating gas temperatures in the disk atmospheres, and therefore underpredict the amount of $\mathrm{CO}$ emission.

If instead we take the well-mixed models at face value (i.e., assuming identical gas and dust temperatures), the model gas temperatures can only be modified by adjusting the dust structure parameters. The model temperatures in the outer disk where the $\mathrm{CO}$ emission is generated are primarily set by the size of the disk (i.e., distance from the star), and the amount of flaring.
To reduce the gas temperatures by the factor of $\sim 5$ required to reproduce the observations for standard $\mathrm{CO} / \mathrm{H}_{2}$ and gas-to-dust conversions, we would need to increase the emitting area $\left(R_{c}\right)$ by roughly an order of magnitude or decrease the scale height $(H)$ to an extreme, flat-disk level: these possibilities are inconsistent with the millimeter visibilities and IR SED, respectively. Given these difficulties, a more straightforward interpretation of the faint line emission in these disks is that the $\mathrm{CO}$ abundances are substantially lower than would be expected for the standard gas-to-dust mass ratio of 100 and/or $\mathrm{CO}$ abundance relative to $\mathrm{H}_{2}$ of $10^{-4}$ (see also Qi et al. 2004; Hughes et al. 2009).

In the absence of measurements of the $\mathrm{H}_{2}$ content of the outer disk, it is not possible to determine whether the lower $\mathrm{CO}$ content results from a reduction in the gas-to-dust mass ratio or abundance of $\mathrm{CO}$ relative to $\mathrm{H}_{2}$, but in either case it marks a significant departure from standard assumptions. Because the rms in the ASTE spectrum of RX J1852.3-3700 is larger than that of RX J1842.9-3532, the upper limit on the CO content of the disk is similarly $\sim 8 \%$ of the initial value, assuming a standard gas-to-dust ratio and $\mathrm{CO}$ abundance. The model with reduced $\mathrm{CO}$ content relative to standard assumptions is also consistent with the limits on $\mathrm{CO}(2-1)$ emission from the SMA. We use RATRAN to generate a sky-projected $\mathrm{CO}(2-1)$ emission map, which is then sampled with the Fourier components of the SMA data using the MIRIAD task uvmodel to account for spatial filtering effects. The model with standard $\mathrm{CO}$ abundance should be detected by the SMA observations, whereas the model with $8 \% \mathrm{CO}$ content is consistent with the upper limits on the $\mathrm{CO}(2-1)$ emission from both systems.

If the gas disk were truncated relative to the dust disk, this could contribute to the low CO content. It is unlikely, however, that both systems would undergo truncation-especially given the dearth of companions within 6" (Köhler et al. 2008)—and the truncation would have to be severe in order to account for an order of magnitude reduction in $\mathrm{CO}$ content. It should also be noted that the conclusion of reduced $\mathrm{CO}$ content is largely independent of the model parameters describing the inner disk and the transitional region between the inner and outer disks. The $\mathrm{CO}(3-2)$ emission arises only from the cold outer disk, and the gas-to-dust ratio is derived only for this region, since we have no information on the gas content of the inner disks of these systems. The extent of the outer disk and its total dust mass are derived from two observational parameters: the millimeter flux and size scale indicated by the resolved visibilities. While the vertical structure and inner disk properties can affect the temperature of the outer disk and therefore the magnitude of the $\mathrm{CO}(3-2)$ emission, these effects are secondary to the basic midplane temperature structure determined by the radial scale of the dust disk. The reduced $\mathrm{CO}$ content is therefore robust to variations in the details of the inner disk structure, since variations in inner disk properties will have only second-order effects on the gross outer disk properties from which this conclusion is derived.

Both systems therefore appear to have undergone a reduction in molecular gas content relative to the standard assumptions for primordial disks. Given their age and transitional SEDs, this may indicate that gas dispersal is underway simultaneously with dust clearing from the inner disk.

\section{DISCUSSION}

We have generated models that can reproduce simultaneously the basic observational diagnostics of the gas and dust disks around RX J1842.9-3532 and RX J1852.3-3700, 
including their broadband SEDs, IRS spectra, resolved millimeter-wavelength visibilities, and $\mathrm{CO}(3-2)$ spectra. As indicated by the mid-IR flux deficit, both systems are transitional, with an inner cavity of significantly decreased dust optical depth.

The disk around RX J1842.9-3532 also exhibits a substantial near-IR excess over the stellar photosphere. It shares this feature with the sample of objects labeled gapped, or "pre-transitional" by Espaillat et al. (2007). They model such systems with an optically thin inner disk bracketed by an optically thick ring close to the star and the optically thick outer disk at large radii. Similar models for the $\mathrm{LkCa} 15$ system, refined with the addition of radiative transfer through the inner disk, are described in Mulders et al. (2010). Isella et al. (2009) modeled the near-IR excess and mid-IR deficit in the LkCa 15 system using a density distribution that increases with distance from the star, but includes a puffed-up inner rim at the dust disk edge. In our study, the inner disk model retains the continuous surface density profile of the outer disk (decreasing with distance from the star), suppressed by the factor $\delta_{\text {cav }}$ (see Figure 5), with no change in scale height at the inner edge of the inner disk. Due to the relatively small scale heights in the inner disk, we can approximate the optical depth to starlight as the product of surface density and $1 \mu \mathrm{m}$ opacity, $\Sigma_{\mathrm{R}} \kappa_{1 \mu \mathrm{m}}$. In this approximation, the cavity is optically thick between 0.01 and $\sim 0.2$ AU but optically thin between $\sim 0.2$ and $5 \mathrm{AU}$, comparable to the models described in Espaillat et al. (2007). These results suggest that transition disks with near-IR excess are not necessarily "gapped" in terms of their surface density or discontinuous in terms of their scale height, since we demonstrate that the inner disk can be modeled using a single, continuous surface density function for the disk cavity that contains just enough mass to have both optically thick and optically thin regimes. Effectively, this indicates that we can place no constraint on the contrast in surface density or scale height between the "gap" and the optically thick ring near the star based on the morphology of the IRS spectrum. This is reflected by the success of several very different models of inner disk structure (Espaillat et al. 2007; Isella et al. 2009; Mulders et al. 2010, this work) in reproducing the characteristic mid-IR deficit surrounded by near- and far-IR excesses.

The model parameters for the two disks discussed in this paper are consistent with those of the nine disks in Ophiuchus that were studied using this method by Andrews et al. (2009). This is perhaps unsurprising, since these targets were similarly selected on the basis of their large submillimeter fluxes. RX J1842.9-3532 and RX J1852.3-3700 have slightly lower masses due to missing material in the otherwise dense disk center, as for the transitional systems in the high-resolution Ophiuchus sample. Yet as with the other transition disks in Andrews et al. (2009) they are still on the high end of the distribution of masses of Taurus and Ophiuchus disks in the sample of Andrews \& Williams (2005, 2007). The transition disk frequency for very low-mass stars in the CrA region has been examined by Sicilia-Aguilar et al. (2008) and Ercolano et al. (2009), with estimates ranging from $15 \%$ to $50 \%$. On the whole, the disks around RX J1842.9-3532 and RX J1852.3-3700 appear comparable to transition disks in other low-mass star-forming regions.

While relatively little is known about the gas evolution of circumstellar disks, it is somewhat surprising that such massive dusty disks should have such low CO content. It is interesting to note that other transitional systems around $\mathrm{K}$ stars have also been found to have low $\mathrm{CO}$ content through similar modeling approaches to that presented here: TW Hya (Qi et al. 2004) and GM Aur (Hughes et al. 2009) have derived CO abundances of $10^{-5}$ and $10^{-6}$, respectively (see also Kastner et al. 1997). The low gas content of TW Hya has recently been corroborated by Herschel observations that appear to indicate a gas-to-dust mass ratio of $\sim 2.6-26$, substantially lower than the standard value of 100 derived for the ISM (Thi et al. 2010). In the absence of knowledge about the $\mathrm{H}_{2}$ content of the system, this is consistent with the Qi et al. (2004) measurement of low CO abundance, which assumed a gas-to-dust mass ratio of 100 . To be clear, the inferred $\mathrm{CO}$ content of these systems is judged to be "low" in comparison with the standard assumptions of a gas-to-dust mass ratio of 100 and a $\mathrm{CO}$ abundance relative to $\mathrm{H}_{2}$ of $10^{-4}$. A reduction in either total molecular gas content (i.e., gas dispersal) or $\mathrm{CO}$ relative to $\mathrm{H}_{2}$ (e.g., molecular depletion) could be responsible, or the standard assumptions could be incorrect. While apparently consistent with ISM conditions, there are almost no observational constraints on either gas-todust or CO-to- $\mathrm{H}_{2}$ ratios in the outer regions of primordial (nontransitional) protoplanetary disks. It is particularly important to understand depletion, since molecular depletion onto dust grains has been suggested as a possible cause of low molecular gas content in circumstellar disks (see, e.g., Dutrey et al. 1997). Depletion is sensitive to the details of the temperature and density structure of the disk, which may be difficult to determine at low spatial resolution. The high-resolution survey of Andrews et al. (2009) finds CO abundances consistent with standard assumptions to within a factor of a few for the three Ophiuchus disks exhibiting strong $\mathrm{CO}$ emission, although they note that most of the sources in the sample are substantially contaminated by the local molecular cloud within which the stars are embedded. Comparable modeling efforts for solartype systems less embedded in their natal clouds are therefore highly desirable for determining the implications of the low $\mathrm{CO}$ content of these transitional systems.

Assuming that the standard assumptions are correct for protoplanetary disks, it is interesting to consider the implications of low CO content for the physical processes clearing the cavities in transitional systems. Among the several mechanisms proposed to clear the central regions of disks, the most popularly invoked are gravitational interaction with a giant planet in formation (e.g., Lin \& Papaloizou 1986; Bryden et al. 1999) and photoevaporation by energetic radiation from the star (e.g., Clarke et al. 2001; Alexander et al. 2006). Many of the dust properties of transitional systems, including the disk masses inferred from dust flux alone and the sharp transition between the inner and outer disks from SED modeling, point toward planet formation as the most likely process. The mass accretion rates of the observed systems, particularly in combination with the disk masses from dust, are also generally more consistent with planet formation as the dominant physical mechanism (see, e.g., discussions in Najita et al. 2007; Alexander \& Armitage 2007). RX J1852.3-3700 in particular occupies an interesting and underpopulated region of the $\dot{M}-M_{*}$ plane investigated by Najita et al. (2007), due to its extremely low accretion rate measurement. The identification of gapped or "pre-transitional" systems like RX J1842.9-3532 has also been interpreted as being more consistent with planet formation than photoevaporative clearing (e.g., Espaillat et al. 2007). It is worth noting, however, that a reduced gas mass for transitional systems relative to protoplanetary disks would present some difficulty for the planet formation scenario, since there is no reason a priori to expect the formation of giant planets to alter the molecular gas content of the outer disk. The masses in Table 1 may be 
misleading, since they represent the total mass in gas and dust of the RADMC model assuming a standard gas-to-dust mass ratio of 100, without taking into account the evidence for low CO content described in Section 4.3; if the low CO content is a result of reduced gas-to-dust mass ratio, the disk masses may be reduced by an order of magnitude or more, placing them squarely within the region of parameter space preferred by photoevaporative clearing models. If this were generally true of transition disks (including TW Hya and GM Aur), it could also provide an alternative explanation for the trend in mass accretion rate as a function of disk mass noted by Najita et al. (2007), since an order of magnitude difference in total mass between the protoplanetary and transition disks would bring the two populations into agreement.

Photoevaporation of disk material by energetic stellar radiation can similarly account for many, but not all, features of these systems. Its most obvious success is in explaining the low $\mathrm{CO}$ content of the outer disk, which cannot easily be accomplished within the framework of planet formation. One complicating factor, however, is the measured accretion rate of material onto the stars based on modeling of the $\mathrm{H} \alpha$ profiles by Pascucci et al. (2007). They calculate an accretion rate of $1 \times 10^{-9} M_{\odot} \mathrm{yr}^{-1}$ for RX J1842.9-3532 and 5 $\times 10^{-10} M_{\odot} \mathrm{yr}^{-1}$ for RX J1852.3-3700. These are roughly an order of magnitude below the average for 1 Myr old stars in Taurus (Gullbring et al. 1998; Calvet et al. 2004), which is consistent with the trend for transitional systems in Taurus noted by Najita et al. (2007). RX J1852.3-3700 in particular occupies an underpopulated region of the $\dot{M}-M_{*}$ plane investigated by Najita et al. (2007), due to its extremely low accretion rate measurement, which could indicate the presence of a very massive planet in the disk. This relatively low but measurable accretion rate is inconsistent with the original predictions of most photoevaporation models (see, e.g., Alexander \& Armitage 2007). Recent work by Owen et al. (2010) suggests that accretion rates of this magnitude may in fact be consistent with the predictions of radiation-hydrodynamic models that incorporate both $\mathrm{X}$-ray and extreme-ultraviolet (EUV) photoevaporation; however, they assume a stellar X-ray luminosity far greater than is typically observed, and EUV luminosities of young stars are not yet well determined. Another potential problem is that the $10^{5} \mathrm{yr}$ timescales predicted for photoevaporative clearing are only marginally consistent with a $\sim 10 \%$ transition disk fraction at ages of $10^{6}-10^{7} \mathrm{yr}$. Certainly more observations are needed to understand the physical mechanisms responsible for clearing the inner cavities of transition disks, including observations across a larger sample and a range of ages, to determine the molecular gas content of transitional relative to primordial disks, and constrain the processes responsible for their dissipation.

At least two well-studied systems at ages of $\sim 10 \mathrm{Myr}$ have been identified as candidate transition disks undergoing photoevaporation: the M star HD 100453 (Collins et al. 2009) and the A star 49 Ceti (Hughes et al. 2008a). The former exhibits a strong IR excess indicative of an optically thick outer disk, but with no evidence of accretion and a maximum gas-to-dust ratio of $4: 1$ in the outer disk. The latter exhibits dust properties similar to a debris disk, yet retains an extended optically thin molecular gas disk with an inner hole. The differences between these systems and the CrA transition disks in this paper are striking, and are perhaps indicative of the range of evolutionary paths over which the transition from protoplanetary to debris disk may occur.

\section{SUMMARY AND CONCLUSIONS}

The 10 Myr old systems RX J1842.9-3532 and RX J1852.3-3700 present a rare opportunity to study the latestage evolution of circumstellar disks. We assemble a multiwavelength data set including the broadband SED, Spitzer IRS spectrum, and generate models of the disk structure for each system that can reproduce the features of the dust continuum emission. We infer the presence of an optically thin 16 AU cavity in the disk around RX J1852.3-3700. The near-IR photospheric excess and mid-IR deficit in the SED of RX J1842.9-3532 allow us to infer the presence of an optically thin 4 AU gap in the disk around RX J1842.9-3532, bracketed by an optically thick ring near the central star $(\sim 0.01-0.2$ AU) and an optically thick outer disk. This configuration marks it as a member of the recently identified class of "pre-transitional" objects (Espaillat et al. 2007). Comparison of the dust disk models with spatially unresolved $\mathrm{CO}(3-2)$ spectra from ASTE allows us to infer a molecular gas content roughly an order of magnitude less than that expected for standard assumptions about the gas-to-dust mass ratio and $\mathrm{CO}$ abundance relative to $\mathrm{H}_{2}$. If the standard assumptions are correct for primordial disks, then this low CO content is suggestive that evolution of the gas disk may be underway simultaneously with the dust disk dispersal.

The transitional systems in CrA observed in this paper therefore mark interesting test cases for distinguishing between proposed mechanisms for gas and dust clearing at late ages. Followup of these objects with instruments at various wavelengths can help to fill in our picture of the properties of the inner and outer disks. For example, the far superior spatial resolution of the Atacama Large Millimeter Array (ALMA) will permit vastly improved modeling of the structure of the extended gas and dust disk, as well as providing direct access to the conditions within the cavity, removing ambiguity about surface densities and scale heights in the inner disk. The sensitivity to spectral line emission provided by ALMA and Herschel will yield insight into the gas mass and chemistry and therefore the origin of the reduced $\mathrm{CO}$ content of the outer disk. In the meantime, observations of rovibrational lines can aid in determining the gas content of the warm inner disk, which will aid in distinguishing between proposed clearing mechanisms. Scattered light images would also be useful for constraining the vertical structure of the disks and reducing degeneracies in these initial models. The suite of instruments currently coming online is poised to revolutionize our ability to characterize the physics of individual disks in the compelling transitional stage of evolution.

We thank C. P. Dullemond for access to the two-dimensional version of RADMC. Partial support for this work was provided by NASA Origins of Solar Systems Program Grant NAG5-11777. A.M.H. acknowledges support from a National Science Foundation Graduate Research Fellowship. Support for S.M.A. was provided by NASA through Hubble Fellowship grant HF-01203-A awarded by the Space Telescope Science Institute, which is operated by the Association of Universities for Research in Astronomy, Inc., for NASA, under contract NAS 5-26555. M.R.M. acknowledges the Harvard Origins of Life Initiative, the Smithsonian Astrophysical Observatory, and a NASA TPF Foundation Science Program grant NNG06GH25G (PI: S. Kenyon) for sabbatical support. 


\section{REFERENCES}

Alexander, R. D., \& Armitage, P. J. 2007, MNRAS, 375, 500

Alexander, R. D., Clarke, C. J., \& Pringle, J. E. 2006, MNRAS, 369, 229

Andrews, S. M., Czekala, I., Wilner, D. J., Espaillat, C., Dullemond, C. P., \& Hughes, A. M. 2010, ApJ, 710, 462

Andrews, S. M., \& Williams, J. P. 2005, ApJ, 631, 1134

Andrews, S. M., \& Williams, J. P. 2007, ApJ, 671, 1800

Andrews, S. M., Wilner, D. J., Hughes, A. M., Qi, C., \& Dullemond, C. P. 2009, ApJ, 700, 1502

Bouwman, J., et al. 2008, ApJ, 683, 479

Brown, J. M., Blake, G. A., Qi, C., Dullemond, C. P., \& Wilner, D. J. 2008, ApJ, 675, L109

Brown, J. M., Blake, G. A., Qi, C., Dullemond, C. P., Wilner, D. J., \& Williams, J. P. 2009, ApJ, 704, 496

Brown, J. M., et al. 2007, ApJ, 664, L107

Bryden, G., Chen, X., Lin, D. N. C., Nelson, R. P., \& Papaloizou, J. C. B. 1999, ApJ, 514, 344

Calvet, N., D’Alessio, P., Hartmann, L., Wilner, D., Walsh, A., \& Sitko, M. 2002, ApJ, 568, 1008

Calvet, N., Muzerolle, J., Briceño, C., Hernández, J., Hartmann, L., Saucedo, J. L., \& Gordon, K. D. 2004, AJ, 128, 1294

Calvet, N., et al. 2005, ApJ, 630, L185

Carpenter, J. M., Wolf, S., Schreyer, K., Launhardt, R., \& Henning, T. 2005, AJ, 129,1049

Carpenter, J. M., et al. 2008, ApJS, 179, 423

Cieza, L., et al. 2007, ApJ, 667, 308

Clarke, C. J., Gendrin, A., \& Sotomayor, M. 2001, MNRAS, 328, 485

Collins, K. A., et al. 2009, ApJ, 697, 557

Cortes, S. R., Meyer, M. R., Carpenter, J. M., Pascucci, I., Schneider, G., Wong, T., \& Hines, D. C. 2009, ApJ, 697, 1305

Currie, T., Lada, C. J., Plavchan, P., Robitaille, T. P., Irwin, J., \& Kenyon, S. J 2009, ApJ, 698, 1

Currie, T., \& Sicilia-Aguilar, A. 2010, ApJ, submitted

D’Alessio, P., Calvet, N., Hartmann, L., Franco-Hernández, R., \& Servín, H. 2006, ApJ, 638, 314

Dullemond, C. P., \& Dominik, C. 2004a, A\&A, 417, 159

Dullemond, C. P., \& Dominik, C. 2004b, A\&A, 421, 1075

Dutrey, A., Guilloteau, S., \& Guelin, M. 1997, A\&A, 317, L55

Ercolano, B., Clarke, C. J., \& Robitaille, T. P. 2009, MNRAS, 394, L141

Espaillat, C., Calvet, N., D’Alessio, P., Hernández, J., Qi, C., Hartmann, L., Furlan, E., \& Watson, D. M. 2007, ApJ, 670, L135

Espaillat, C., Calvet, N., Luhman, K. L., Muzerolle, J., \& D’Alessio, P. 2008, ApJ, 682, L125

Espaillat, C., et al. 2010, ApJ, 717, 441

Gullbring, E., Hartmann, L., Briceno, C., \& Calvet, N. 1998, ApJ, 492, 323

Hartmann, L., Calvet, N., Gullbring, E., \& D’Alessio, P. 1998, ApJ, 495, 385
Hogerheijde, M. R., \& van der Tak, F. F. S. 2000, A\&A, 362, 697

Hughes, A. M., Wilner, D. J., Calvet, N., D’Alessio, P., Claussen, M. J., \& Hogerheijde, M. R. 2007, ApJ, 664, 536

Hughes, A. M., Wilner, D. J., Kamp, I., \& Hogerheijde, M. R. 2008a, ApJ, 681, 626

Hughes, A. M., Wilner, D. J., Qi, C., \& Hogerheijde, M. R. 2008b, ApJ, 678 1119

Hughes, A. M., et al. 2009, ApJ, 698, 131

Isella, A., Carpenter, J. M., \& Sargent, A. I. 2009, ApJ, 701, 260

Kamp, I., \& Dullemond, C. P. 2004, ApJ, 615, 991

Kastner, J. H., Zuckerman, B., Weintraub, D. A., \& Forveille, T. 1997, Science, 277,67

Kim, J. S., et al. 2005, ApJ, 632, 659

Köhler, R., Neuhäuser, R., Krämer, S., Leinert, C., Ott, T., \& Eckart, A. 2008, A\&A, 488, 997

Lay, O. P., Carlstrom, J. E., \& Hills, R. E. 1997, ApJ, 489, 917

Lin, D. N. C., \& Papaloizou, J. 1986, ApJ, 309, 846

Lynden-Bell, D., \& Pringle, J. E. 1974, MNRAS, 168, 603

Meyer, M. R., et al. 2006, PASP, 118, 1690

Mulders, G. D., Dominik, C., \& Min, M. 2010, A\&A, 512, A11

Muzerolle, J., Allen, L. E., Megeath, S. T., Hernández, J., \& Gutermuth, R. A. 2010, ApJ, 708, 1107

Najita, J. R., Strom, S. E., \& Muzerolle, J. 2007, MNRAS, 378, 369

Neuhäuser, R., et al. 2000, A\&AS, 146, 323

Ossenkopf, V., \& Henning, T. 1994, A\&A, 291, 943

Owen, J. E., Ercolano, B., Clarke, C. J., \& Alexander, R. D. 2010, MNRAS, 401, 1415

Pascucci, I., et al. 2007, ApJ, 663, 383

Piétu, V., Dutrey, A., \& Guilloteau, S. 2007, A\&A, 467, 163

Qi, C., Wilner, D. J., Calvet, N., Bourke, T. L., Blake, G. A., Hogerheijde, M. R., Ho, P. T. P., \& Bergin, E. 2006, ApJ, 636, L157

Qi, C., et al. 2004, ApJ, 616, L11

Sicilia-Aguilar, A., Henning, T., \& Hartmann, L. W. 2010, ApJ, 710, 597

Sicilia-Aguilar, A., Henning, T., Juhász, A., Bouwman, J., Garmire, G., \& Garmire, A. 2008, ApJ, 687, 1145

Silverstone, M. D., et al. 2006, ApJ, 639, 1138

Skrutskie, M. F., Dutkevitch, D., Strom, S. E., Edwards, S., Strom, K. M., \& Shure, M. A. 1990, AJ, 99, 1187

Skrutskie, M. F., et al. 2006, AJ, 131, 1163

Strom, K. M., Strom, S. E., Edwards, S., Cabrit, S., \& Skrutskie, M. F. 1989, AJ, 97, 1451

Thi, W. F., van Dishoeck, E. F., Pontoppidan, K. M., \& Dartois, E. 2010, arXiv: 1002.2157

Uzpen, B., Kobulnicky, H. A., Semler, D. R., Bensby, T., \& Thom, C. 2008, ApJ, 685,1157

Wang, Y., Jaffe, D. T., Graf, U. U., \& Evans, II, N. J. 1994, ApJS, 95, 503

Woitke, P., Kamp, I., \& Thi, W. 2009, A\&A, 501, 383 